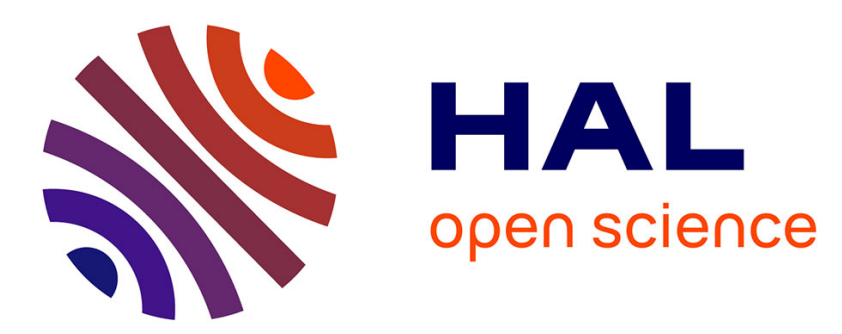

\title{
Approximation of Liquid-Vapor Phase Transition for Compressible Fluids with Tabulated EOS
}

\author{
Gloria Faccanoni, Samuel Kokh, Grégoire Allaire
}

\section{To cite this version:}

Gloria Faccanoni, Samuel Kokh, Grégoire Allaire. Approximation of Liquid-Vapor Phase Transition for Compressible Fluids with Tabulated EOS. Comptes Rendus de l'Academie des Sciences. Série IV, Physique, Astronomie, 2010, 348 (7-8), pp.473-478. 10.1016/j.crma.2010.01.012 . hal-00976950

\section{HAL Id: hal-00976950 \\ https://hal-univ-tln.archives-ouvertes.fr/hal-00976950}

Submitted on 3 Dec 2021

HAL is a multi-disciplinary open access archive for the deposit and dissemination of scientific research documents, whether they are published or not. The documents may come from teaching and research institutions in France or abroad, or from public or private research centers.
L'archive ouverte pluridisciplinaire HAL, est destinée au dépôt et à la diffusion de documents scientifiques de niveau recherche, publiés ou non, émanant des établissements d'enseignement et de recherche français ou étrangers, des laboratoires publics ou privés. 


\title{
Approximation of liquid-vapor phase transition for compressible fluids with tabulated EOS
}

\section{Approximation du changement de phase dynamique avec des lois d'état tabulées}

\author{
Gloria Faccanoni ${ }^{a}$, Samuel Kokh ${ }^{b}$, Grégoire Allaire ${ }^{c}$ \\ a IMATH - université du Sud Toulon-Var, avenue de l'université, 83957 La Garde cedex, France \\ b DEN/DANS/DM2S/SFME/LETR, Commissariat à l'énergie atomique, Saclay, 91191 Gif-sur-Yvette cedex, France \\ c CMAP, École polytechnique, CNRS, 91128 Palaiseau cedex, France
}

\begin{abstract}
A B S T R A C T
This Note investigates the approximation of phase change in compressible fluids with complex equation of state (EOS). Assuming a local and instantaneous equilibrium with respect to phasic pressures, temperatures and chemical potentials when both phases are present leads to the classical definition of an equilibrium EOS for the two-phase medium. Unfortunately, there is no explicit expression of the equilibrium EOS in most cases. We propose simple means to approximate the equilibrium EOS when both phases are governed by very general EOS, including tabulated ones. We present a relaxation type numerical algorithm based on this approximation for simulating two-phase flows with phase change.

(c) 2010 Published by Elsevier Masson SAS on behalf of Académie des sciences.
\end{abstract}

\section{R É S U M É}

Cette Note étudie l'approximation des transitions de phase pour des fluides compressibles munis de lois d'état complexes. En postulant un équilibre instantané et local des pressions, températures et potentiels chimiques de chaque phase, lorsqu'elles sont toutes deux présentes, on définit classiquement une équation d'état à l'équilibre pour le milieu diphasique. Malheureusement, il n'y a pas d'expression explicite pour la loi à l'équilibre dans le cas général. Nous proposons ici une méthode simple pour approcher cette loi d'état lorsque les propriétés des deux phases sont décrites par des lois très générales, éventuellement sous forme tabulée. Enfin, nous présentons un schéma numérique articulé autour d'une méthode de relaxation se basant sur la définition de cette approximation de l'équilibre afin de simuler des écoulements diphasiques avec changement de phase.

(c) 2010 Published by Elsevier Masson SAS on behalf of Académie des sciences.

\section{Version française abrégée}

Nous proposons dans cette Note un moyen simple de simuler des phénomènes de changement de phase liquide-vapeur dynamiques en utilisant des lois thermodynamiques quelconques, par exemple tabulées. Le cadre que nous adoptons est celui d'un modèle diphasique et d'une méthode de relaxation basée sur une approche Volumes Finis [2,6,7]. Nous supposons que nous sommes loin du point critique, ainsi pour le système diphasique qui nous intéresse, chaque phase est considérée

E-mail addresses: gloria.faccanoni@univ-tln.fr (G. Faccanoni), samuel.kokh@cea.fr (S. Kokh), allaire@cmap.polytechnique.fr (G. Allaire).

URLs: http://faccanoni.univ-tln.fr (G. Faccanoni), http://www.cmap.polytechnique.fr/ allaire (G. Allaire). 
comme un fluide compressible muni de sa propre loi d'état. Ces dernières sont données sous la forme $\left(\tau_{\alpha}, \varepsilon_{\alpha}\right) \mapsto s_{\alpha}$, où $\tau_{\alpha}>0, \varepsilon_{\alpha}>0$ et $s_{\alpha}$ sont respectivement le volume spécifique, l'énergie interne spécifique et l'entropie spécifique de la phase vapeur $\alpha=v$ (resp. liquide $\alpha=l$ ). On suppose que la matrice hessienne de $s_{\alpha}$ est toujours définie négative et nous définissons de manière classique la température $T_{\alpha}=1 /\left(\partial s_{\alpha} / \partial \varepsilon_{\alpha}\right)_{\tau_{\alpha}}>0$, la pression $P_{\alpha}=T_{\alpha} \cdot\left(\partial s_{\alpha} / \partial \tau_{\alpha}\right)_{\varepsilon_{\alpha}}>0$ et le potentiel chimique $g_{\alpha}=\varepsilon_{\alpha}+P_{\alpha} \tau_{\alpha}-T_{\alpha} s_{\alpha}$ de la phase $\alpha=l, v$ [5]. La fraction de masse de la phase $\alpha=v$ (resp. $\alpha=l$ ) est notée $y_{v}=y$ (resp. $y_{l}=1-y$ ) et nous supposons que $y \in[0,1]$. La densité du milieu $\varrho$ et l'énergie interne spécifique du milieu $\varepsilon$ sont définies par $\varrho^{-1}=\tau=y \tau_{v}+(1-y) \tau_{l}$ et $\varepsilon=y \varepsilon_{v}+(1-y) \varepsilon_{l}$. Le transfert de masse entre les phases est modélisé en dotant le système d'une entropie, dite "entropie d'équilibre », notée $(\tau, \varepsilon) \mapsto s^{\text {eq }}$ et définie par l'expression (1). Ceci revient à imposer pour le milieu, dès que cela est possible, un équilibre en pression, température et potentiel chimique entre les deux phases. Si on néglige les effets capillaires, les effets visqueux et la diffusion thermique, alors le système étudié se ramène aux équations d'Euler pour un fluide compressible muni d'une loi de pression définie par $P^{\mathrm{eq}}=\left(\partial s^{\mathrm{eq}} / \partial \tau\right)_{\varepsilon} /\left(\partial s^{\mathrm{eq}} / \partial \varepsilon\right)_{\tau}$. Le système ainsi formé est strictement hyperbolique sous des hypothèses simples [2] et s'écrit sous la forme (2). Bien que la définition de la loi d'état équilibre soit cohérente sous des hypothèse standards $[2,6,10,13,14]$, son évalution rend la discrétisation de (2) délicate. Il s'agit en effet d'estimer les valeurs de $\tau_{\alpha}, \varepsilon_{\alpha}, \alpha=l, v$ qui maximisent $y s_{v}\left(\tau_{v}, \varepsilon_{v}\right)+(1-y) s_{l}\left(\tau_{l}, \varepsilon_{l}\right)$. Néanmoins une approche par relaxation en deux étapes, un pas de convection suivi d'un pas de projection, permet de confiner ce calcul dans l'étape de projection. Cette stratégie a été mise en œuvre dans [8-10] pour des lois d'état du type gaz parfait et dans [7] pour le cas de deux phases modélisées par des équations d'état de type Stiffened Gas. Nous proposons ici une extension de cette méthode pour des lois d'état quelconques, forcément définies de manière analytique, et par exemple tabulées à partir de valeurs expérimentales. Soit $\mathcal{C}=\{\tau>0, \varepsilon>0\}$ l'ensemble des états admissibles et un couple donné $(\tau, \varepsilon) \in \mathcal{C}$. Le calcul des variables phasiques à l'équilibre peut être décrit grâce à l'alternative suivante : soit il existe un état pour lequel les relations d'équilibre (4)-(5) sont vérifiées et dans ce cas les deux phases sont dite "à saturation"; soit le milieu est localement monophasique. Lorsque l'on est à saturation, il est bien connu que l'état $\left(\tau_{v}, \tau_{l}, \varepsilon_{v}, \varepsilon_{l}, y\right)$ du milieu peut être paramétré grâce à une seule variable thermodynamique comme $P$ ou $T$. Ceci définit la courbe de coexistence $T \mapsto P^{\text {sat }}(T)$. Notons $\tau_{\alpha}^{\text {sat }}(T)=\tau_{\alpha}\left(T, P^{\text {sat }}(T)\right)$ et $\varepsilon_{\alpha}^{\text {sat }}(T)=\varepsilon_{\alpha}\left(T, P^{\text {sat }}(T)\right), \alpha=l, v$, dont les valeurs mesurées experimentalement sont souvent disponibles pour de nombreux fluides [12]. Nous montrons que la résolution du système (4) peut se ramener à déterminer $T$ vérifiant la relation implicite (6) avec $(A, B, C, D)(T)=$ $\left(1 / \tau_{v}^{\text {sat }}, \tau_{l}^{\text {sat }} / \tau_{v}^{\text {sat }}, 1 / \varepsilon_{v}^{\text {sat }}, \varepsilon_{l}^{\text {sat }} / \varepsilon_{v}^{\text {sat }}\right)(T)$. Ensuite, au lieu de résoudre (6), nous proposons de chercher $T$ solution de (7) où $T \mapsto(\hat{A}, \hat{B}, \hat{C}, \hat{D})(T)$ sont des approximations convenables pour les lois tabulées $T \mapsto(A, B, C, D)(T)$. Nous fournirons un exemple de telles approximations pour l'eau dans la plage de valeurs $T \in[281,583] \mathrm{K}$ en annexe. Nous concluons par une simulation numérique bidimensionnelle qui illustre la capacité du modèle et de la méthode numérique à prendre en compte le changement de phase dans le cas de lois tabulées.

\section{Introduction: The dynamical liquid-vapor phase change model}

In this Note we propose an approximation method for using general equations of state (EOS), including tabulated ones, in the numerical simulation of dynamical liquid-vapor phase change [2,6,7]. Our paper is structured as follows: first we recall the definition of our equilibrium two-phase system. The equilibrium thermodynamical variables are obtained through an implicit non-linear system that we shall exhibit. Then, we introduce an approximate non-linear equation which allows us to cope with tabulated laws. Finally we present a set of fitted interpolation functions for liquid-vapor water and a twodimensional numerical simulation showing that the model and the numerical scheme can reproduce cavitation mechanisms with tabulated EOS for water and steam.

\subsection{Characterization of the two-phase medium}

We briefly recall the hypotheses that define the two-phase medium studied in $[2,6,7]$. We suppose that the medium is far from the critical point. Therefore both phases are modelled by compressible fluids equipped with an EOS given by a function $\left(\tau_{\alpha}, \varepsilon_{\alpha}\right) \mapsto s_{\alpha}$, where $\tau_{\alpha}>0, \varepsilon_{\alpha}>0$ and $s_{\alpha}$ denote respectively the specific volume, the specific internal energy and the specific entropy of the liquid phase $\alpha=l$ (resp. vapor phase $\alpha=v$ ). The Hessian matrix of $s_{\alpha}$ is always negative definite and we define classically the temperature $T_{\alpha}=1 /\left(\partial s_{\alpha} / \partial \varepsilon_{\alpha}\right)_{\tau_{\alpha}}>0$, the pressure $P_{\alpha}=T_{\alpha} \cdot\left(\partial s_{\alpha} / \partial \tau_{\alpha}\right)_{\varepsilon_{\alpha}}>0$ and the chemical potential $g_{\alpha}=\varepsilon_{\alpha}+P_{\alpha} \tau_{\alpha}-T_{\alpha} s_{\alpha}$ of the phase $\alpha=l, v$. The mass fraction of the phase $\alpha=v$ (resp. $\alpha=l$ ) is noted $y_{v}=y$ (resp. $y_{l}=1-y$ ) and we suppose $y \in[0,1]$. The total density and specific internal energy are classically defined respectively by $\varrho^{-1}=\tau=y \tau_{v}+(1-y) \tau_{l}$ and $\varepsilon=y \varepsilon_{v}+(1-y) \varepsilon_{l}$. The model of mass transfer examined in $[2,6,7]$ relies on the classical assumption that there is an instantaneous equilibrium at each point of the domain with respect to pressure, temperature and chemical potential between both phases. Under classical thermodynamical assumptions that we shall not detail here (see e.g. $[2-6,8,9]$ ), this hypotheses boils down to provide the two-phase material with an equilibrium EOS defined thanks to the equilibrium entropy denoted by $(\tau, \varepsilon) \mapsto s^{\mathrm{eq}}$ and given by

$$
s^{\mathrm{eq}}(\tau, \varepsilon)=\sup \left\{y s_{v}\left(\tau_{v}, \varepsilon_{v}\right)+(1-y) s_{l}\left(\tau_{l}, \varepsilon_{l}\right) \mid \tau=y \tau_{v}+(1-y) \tau_{l}, \varepsilon=y \varepsilon_{v}+(1-y) \varepsilon_{l}, 0<y<1\right\} .
$$

The pressure $(\tau, \varepsilon) \mapsto P^{\mathrm{eq}}$ for the two-phase medium can then be defined by $P^{\mathrm{eq}}=\left(\partial s^{\mathrm{eq}} / \partial \tau\right)_{\varepsilon} /\left(\partial s^{\mathrm{eq}} / \partial \varepsilon\right)_{\tau}$. Finally, if we suppose that both phases have the same velocity $\mathbf{u}$ in the two-phase medium and if we neglect all dissipative phenomena and local effects such as surface tension, gravity or heat diffusion, the two-phase system that governs the phase change 
phenomena consists of the compressible Euler equations with the equilibrium pressure law $(\tau, \varepsilon) \mapsto P^{\mathrm{eq}}(\tau, \varepsilon)$, namely $[2,6,7]$ :

$$
\begin{aligned}
& \partial_{t} \varrho+\operatorname{div}(\varrho \mathbf{u})=0, \quad \partial_{t}(\varrho \mathbf{u})+\operatorname{div}\left(\varrho \mathbf{u} \otimes \mathbf{u}+P^{\mathrm{eq}} \mathrm{Id}\right)=\mathbf{0}, \\
& \partial_{t}\left(\varrho \varepsilon+\varrho|\mathbf{u}|^{2} / 2\right)+\operatorname{div}\left[\left(\varrho \varepsilon+\varrho|\mathbf{u}|^{2} / 2+P^{\mathrm{eq}}\right) \mathbf{u}\right]=0 .
\end{aligned}
$$

Some mathematical properties of system (2) are discussed in [2,6,10,13,14]: it is strictly hyperbolic under simple hypothesis concerning the pure phases EOS [2]. A delicate matter when discretizing the system (2) resides in computing variables obtained through the equilibrium EOS as for example the value of the pressure. This issue was addressed in [2,6,7] thanks to a two-step convection/projection relaxation numerical solver. This method allows one to decouple computation of the equilibrium thermodynamical parameters from the approximation of the convection by confining it in the projection step. The implementation proposed in [7] was only dealing with the case when both phases are described by stiffened gas EOS. Our purpose is here to extend this strategy for tabulated thermodynamical laws.

\subsection{A two-step convection/projection relaxation numerical solver}

Following similar lines as in [7,9], we propose a two-step relaxation method for approximating the solution of the system (2). We consider below the augmented system (3) that is a relaxed version of (2):

$$
\partial_{t} \mathbf{W}+\operatorname{div} \mathbf{F}(\mathbf{W}, z)=\mathbf{R}(\mathbf{W}, z), \quad \partial_{t} z+\mathbf{u} \cdot \operatorname{grad} z=Q(\mathbf{W}, z)
$$

where

$$
\begin{array}{lll}
\mathbf{W}=\left(z \varrho_{v},(1-z) \varrho_{l}, \varrho \mathbf{u}, \varrho\left(\varepsilon+\frac{|\mathbf{u}|^{2}}{2}\right)\right), & \mathbf{F}(\mathbf{W}, z)=\left(z \varrho_{v} \mathbf{u},(1-z) \varrho_{l} \mathbf{u}, \varrho \mathbf{u} \otimes \mathbf{u}+\pi \operatorname{Id},\left(\varrho\left(\varepsilon+\frac{|\mathbf{u}|^{2}}{2}\right)+\pi\right) \mathbf{u}\right), \\
Q(\mathbf{W}, z)=v\left(P_{v}-P_{l}\right) / \vartheta, & \mathbf{R}(\mathbf{W}, z)=\left(\mu\left(g_{l}-g_{v}\right) / \vartheta,-\mu\left(g_{l}-g_{v}\right) / \vartheta, 0,0\right),
\end{array}
$$

with the volume fraction $z$ such that $\varrho=z \varrho_{v}+(1-z) \varrho_{l}$, the pressure $\pi$ such that $\pi=z P_{v}+(1-z) P_{l}$ and the closure $T_{v}=T_{l}=\vartheta$.

Formally the equilibrium $\mu, v \rightarrow+\infty$ implies that $\mathbf{R}(\mathbf{W}, z)=\mathbf{0}, Q(\mathbf{W}, z)=0$ which matches equilibrium relations (4). The discretization of system (2) is a two-step algorithm: step (I) let $(\mathbf{W}, z)^{n}$ be the state variables at $t=t^{n}$; the augmented variable $(\mathbf{W}, z)^{n}$ is updated to $(\mathbf{W}, z)^{n+1 / 2}$ by solving the system (3) with $\mathbf{R}(\mathbf{W}, z)=\mathbf{0}, Q(\mathbf{W}, z)=0$. This step can be achieved thanks to a Finite Volume Roe-type solver proposed in [1] with appropriate interpolations of pure tabulated EOS (as in [11]); step (II) perform an approximated projection of the state $(\mathbf{W}, z)^{n+1 / 2}$ onto the equilibrium defined by $\mu, v \rightarrow+\infty$ by approaching the solution of $\mathbf{R}(\mathbf{W}, z)=\mathbf{0}, Q(\mathbf{W}, z)=0$. This step is achieved by seeking the solution $\hat{T}$ of Eq. (7) (see below), thanks to a dichotomy algorithm. We then set

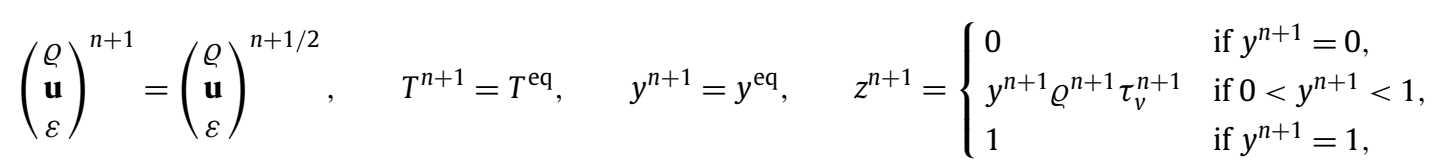

which defines $(\mathbf{W}, z)^{n+1}$. One can see that the mass fraction is updated by the projection step II. This means that the mass transfer is driven by the two-phase equilibrium. Moreover, the model intrinsically accounts for phase appearance and phase disappearance.

\section{Equilibrium EOS: Definition of the "Saturated States"}

We briefly recall here the classical definition of the "Saturated States". Let us denote by $\left(\tau_{v}^{*}, \varepsilon_{v}^{*}, \tau_{l}^{*}, \varepsilon_{l}^{*}, y^{*}\right)$ the maximizer of the mixture entropy $y s_{v}+(1-y) s_{l}$ under the constraints set by definition (1). Let $\mathcal{C}=\{\tau>0, \varepsilon>0\}$. When $0<y^{*}<1$, both phases are present at equilibrium and they are said to be "at saturation". In this case, for a given couple $(\tau, \varepsilon) \in \mathcal{C}$, the saturated state $\left(\tau_{v}^{*}, \varepsilon_{v}^{*}, \tau_{l}^{*}, \varepsilon_{l}^{*}, y^{*}\right)$ is also the unique solution of the following optimality system (4)-(5) for the maximisation (1):

$$
\begin{aligned}
& (\tau, \varepsilon)=y\left(\tau_{v}, \varepsilon_{v}\right)+(1-y)\left(\tau_{l}, \varepsilon_{l}\right), \quad\left(P_{v}, T_{v}, g_{v}\right)\left(\tau_{v}, \varepsilon_{v}\right)=\left(P_{l}, T_{l}, g_{l}\right)\left(\tau_{l}, \varepsilon_{l}\right), \\
& y \in(0,1) .
\end{aligned}
$$

Considering the solution of (4)-(5), if we note $P=P_{v}=P_{l}$ and $T=T_{v}=T_{l}$, then saturated states $\left(\tau_{v}^{*}, \varepsilon_{v}^{*}, \tau_{l}^{*}, \varepsilon_{l}^{*}, y^{*}\right)$ can be parametrized by a single thermodynamical variable such as $P$ or $T$. This classical result $[5,6]$ provides the definition of the coexistence curve $T \mapsto P=P^{\text {sat }}(T)$. Far from the critical point, we can reasonably assume that $\tau_{v}(P, T)>\tau_{l}(P, T)$, for any $P>0$ and $T>0$, without breaking the thermodynamical coherence. Now the overall procedure for computing the equilibrium EOS lies in the following alternative: 
(a)

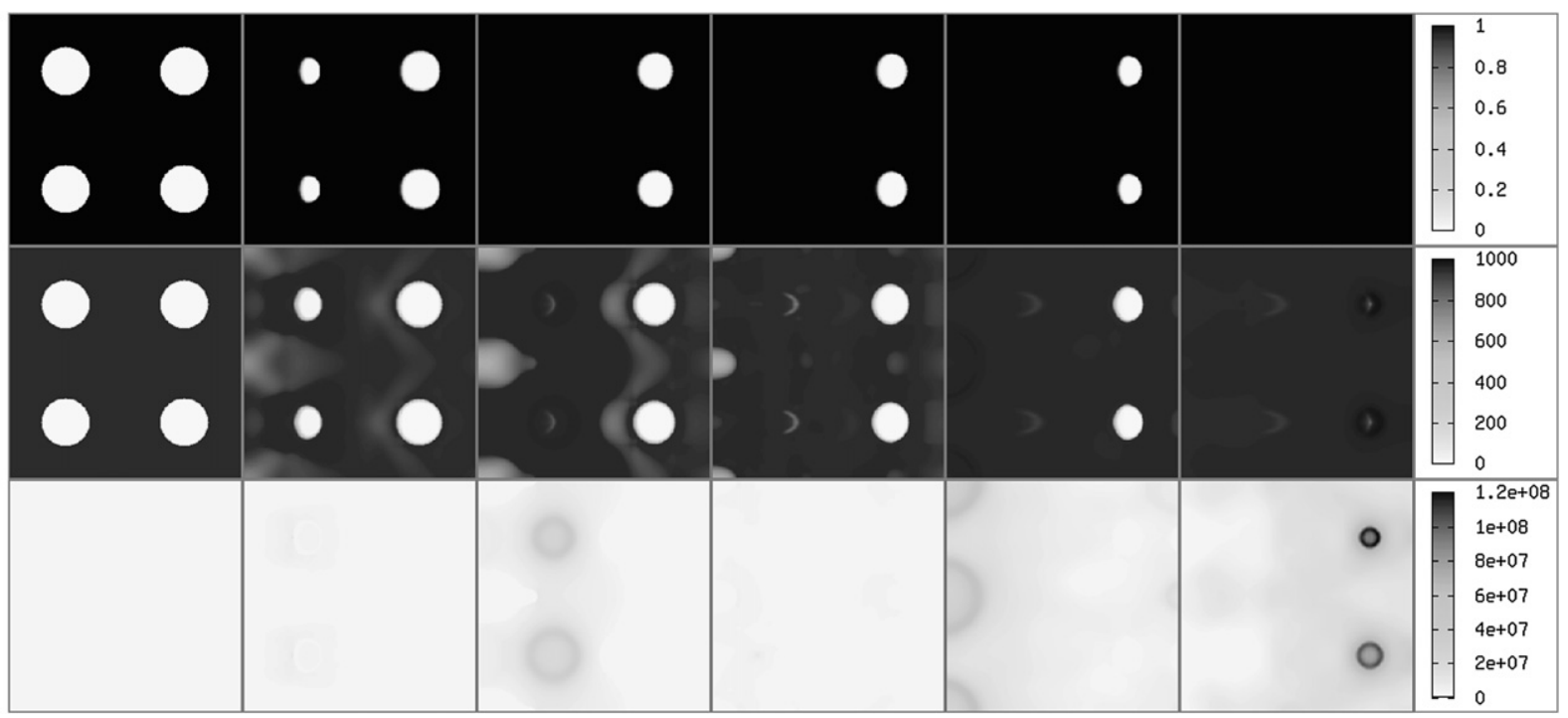

Fig. 1. Evolution of the mass fraction $y$ (a), the total density $\varrho$ (b) and the pressure $P$ (c) for time varying from $t=0$ ms to $t=3.30$ ms.

(i) if $\left(\tau_{v}^{*}, \varepsilon_{v}^{*}, \tau_{l}^{*}, \varepsilon_{l}^{*}, y^{*}\right)$ verifies both (4) and (5) then $y^{*}=\frac{\tau-\tau_{l}^{*}}{\tau_{v}^{*}-\tau_{l}^{*}}$ and we set $P^{\mathrm{eq}}(\tau, \varepsilon)=P_{v}\left(\tau_{v}^{*}, \varepsilon_{v}^{*}\right)=P_{l}\left(\tau_{l}^{*}, \varepsilon_{l}^{*}\right)$, $T^{\mathrm{eq}}(\tau, \varepsilon)=T_{v}\left(\tau_{v}^{*}, \varepsilon_{v}^{*}\right)=T_{l}\left(\tau_{l}^{*}, \varepsilon_{l}^{*}\right), y^{\mathrm{eq}}=y^{*}$

(ii) otherwise, if $s_{v}(\tau, \varepsilon)>s_{l}(\tau, \varepsilon)$ (resp. $s_{v}(\tau, \varepsilon)<s_{l}(\tau, \varepsilon)$ ) then we set $y^{\mathrm{eq}}=1$ and $P^{\mathrm{eq}}(\tau, \varepsilon)=P_{v}(\tau, \varepsilon), T^{\mathrm{eq}}(\tau, \varepsilon)=$ $T_{v}(\tau, \varepsilon)\left(\right.$ resp. $y^{\mathrm{eq}}=0$ and $P^{\mathrm{eq}}(\tau, \varepsilon)=P_{l}(\tau, \varepsilon), T^{\mathrm{eq}}(\tau, \varepsilon)=T_{l}(\tau, \varepsilon)$ ).

The case (ii) corresponds to a pure phase $\alpha=l$ or $\alpha=v$ state which means that either system (4) has no solution either the condition (5) fails. However, we can then see that settling between the case (i) and the case (ii) requires first to solve the non-linear system (4) which is a difficult step, especially when one deals with general thermodynamical functions, a fortiori with tabulated laws. Let us mention that even for general stiffened gas laws, there is no explicit solution for (4) [6]. In this Note we propose a general method that allows one to approximate the possible solution of (4) using experimental data.

Let us note $\tau_{\alpha}^{\text {sat }}(T)=\tau_{\alpha}\left(T, P^{\text {sat }}(T)\right)$ and $\varepsilon_{\alpha}^{\text {sat }}(T)=\varepsilon_{\alpha}\left(T, P^{\text {sat }}(T)\right), \alpha=l, v$, whose values are commonly available in experiment measurements tables [12]. Given a fixed couple $(\tau, \varepsilon) \in \mathcal{C}$, we observe that solving (4) is equivalent to seeking $T$ as the solution of

$$
[\tau A(T)-1][B(T)-1]^{-1}=[\varepsilon C(T)-1][D(T)-1]^{-1},
$$

where $(A, B, C, D)(T)=\left(1 / \tau_{v}^{\text {sat }}, \tau_{l}^{\text {sat }} / \tau_{v}^{\text {sat }}, 1 / \varepsilon_{v}^{\text {sat }}, \varepsilon_{l}^{\text {sat }} / \varepsilon_{v}^{\text {sat }}\right)(T)$ (for details see [6,7]). Eq. (6) is reffered to the Phase Change Equation. For a fixed $(\tau, \varepsilon) \in \mathcal{C}$, instead of solving (6) we propose to seek $T$ as the solution of the alternative equation

$$
[\tau \hat{A}(T)-1][\hat{B}(T)-1]^{-1}=[\varepsilon \hat{C}(T)-1][\hat{D}(T)-1]^{-1},
$$

where $T \mapsto(\hat{A}, \hat{B}, \hat{C}, \hat{D})(T)$ are proper approximations of the tabulated laws $T \mapsto(A, B, C, D)(T)$. Eq. (7) is reffered to the Approximate Phase Change Equation.

We provide in Appendix A an example of approximate functions $T \mapsto(\hat{A}, \hat{B}, \hat{C}, \hat{D})$ for water used for the simulation of the phase transition of Fig. 1. Other suitable approximations for the case of dodecane are available in [6].

\section{A numerical simulation}

In Fig. 1 we present preliminary simulation results of cavitation phenomena. The overall numerical strategy consists in the following fractional step approach: step (I) - solve the off-equilibrium hydrodynamics system using the Roe-type solver proposed in [1] thanks to the approximation of pure phase laws proposed in [11] (i.e. stiffened gas laws fitted on tabulated laws for water and steam [12]); step (II) - project onto the approximate equilibrium thanks to the Approximate Phase Change Equation (7) with the approximate functions proposed in Appendix A.

Our method is tested against the case of a vapor bubble compression. We consider a $1 \mathrm{~m}$ side length $2 \mathrm{D}$ square domain discretized over a $300 \times 300$-cell mesh. Four water vapor bubbles are surrounded by liquid water. The initial temperature is fixed to $T_{0}=439 \mathrm{~K}$ and the fluid is initially at rest in the whole domain. Both phases are supposed to be at saturation at $t=0$. We suppose the left boundary to be a piston that moves towards right at constant speed by imposing a constant velocity $u_{p}=30 \mathrm{~m} / \mathrm{s}$ in the fictitious cells. Other boundary conditions are reflective walls. The moving piston generates a pressure wave that compresses the vapor. As the pressure increases the vapor starts to condensate: the bubbles shrink and disappear. 
Table A.1

Coefficients of the functions $\hat{A}, \hat{B}, \hat{C}$ and $\hat{D}$ for the approximation of water.

\begin{tabular}{|c|c|c|c|c|}
\hline$k$ & $\hat{A}_{k}$ & $\hat{B}_{k}$ & $\hat{C}_{k}$ & $\hat{D}_{k}$ \\
\hline-9 & & $3.436 \times 10^{24}$ & & \\
\hline-8 & & $-3.623 \times 10^{21}$ & & \\
\hline-7 & & $5.197 \times 10^{18}$ & & $4.008 \times 10^{18}$ \\
\hline-6 & & $4.879 \times 10^{16}$ & & $-3.903 \times 10^{16}$ \\
\hline-5 & & $-4.472 \times 10^{13}$ & & $-1.782 \times 10^{14}$ \\
\hline-4 & & $-5.153 \times 10^{11}$ & & $3.695 \times 10^{12}$ \\
\hline-3 & & $-2.059 \times 10^{9}$ & & $-1.387 \times 10^{10}$ \\
\hline-2 & & $1.256 \times 10^{7}$ & & $-1.743 \times 10^{7}$ \\
\hline-1 & $-4.212 \times 10^{3}$ & $2.448 \times 10^{4}$ & & $1.941 \times 10^{5}$ \\
\hline 0 & $-3.172 \times 10^{1}$ & $-1.949 \times 10^{2}$ & $6.724 \times 10^{-7}$ & $1.524 \times 10^{2}$ \\
\hline 1 & $6.078 \times 10^{-1}$ & $5.116 \times 10^{-2}$ & $-0.318 \times 10^{-9}$ & $-4.027 \times 10^{0}$ \\
\hline 2 & $-4.167 \times 10^{-3}$ & $7.768 \times 10^{-4}$ & $1.942 \times 10^{-11}$ & $1.295 \times 10^{-2}$ \\
\hline 3 & $1.751 \times 10^{-5}$ & $1.046 \times 10^{-6}$ & $-6.844 \times 10^{-14}$ & $-1.461 \times 10^{-5}$ \\
\hline 4 & $-4.806 \times 10^{-8}$ & $-8.709 \times 10^{-9}$ & $1.343 \times 10^{-16}$ & $1.079 \times 10^{-8}$ \\
\hline 5 & $8.636 \times 10^{-11}$ & $7.531 \times 10^{-12}$ & $1.385 \times 10^{-19}$ & $4.868 \times 10^{-11}$ \\
\hline 6 & $-9.785 \times 10^{-14}$ & $2.382 \times 10^{-14}$ & $5.957 \times 10^{-22}$ & $-5.168 \times 10^{-13}$ \\
\hline 7 & $6.336 \times 10^{-17}$ & $-6.084 \times 10^{-17}$ & & $1.957 \times 10^{-17}$ \\
\hline 8 & $-1.784 \times 10^{-20}$ & $5.373 \times 10^{-20}$ & & \\
\hline 9 & & $-1.726 \times 10^{-23}$ & & \\
\hline
\end{tabular}

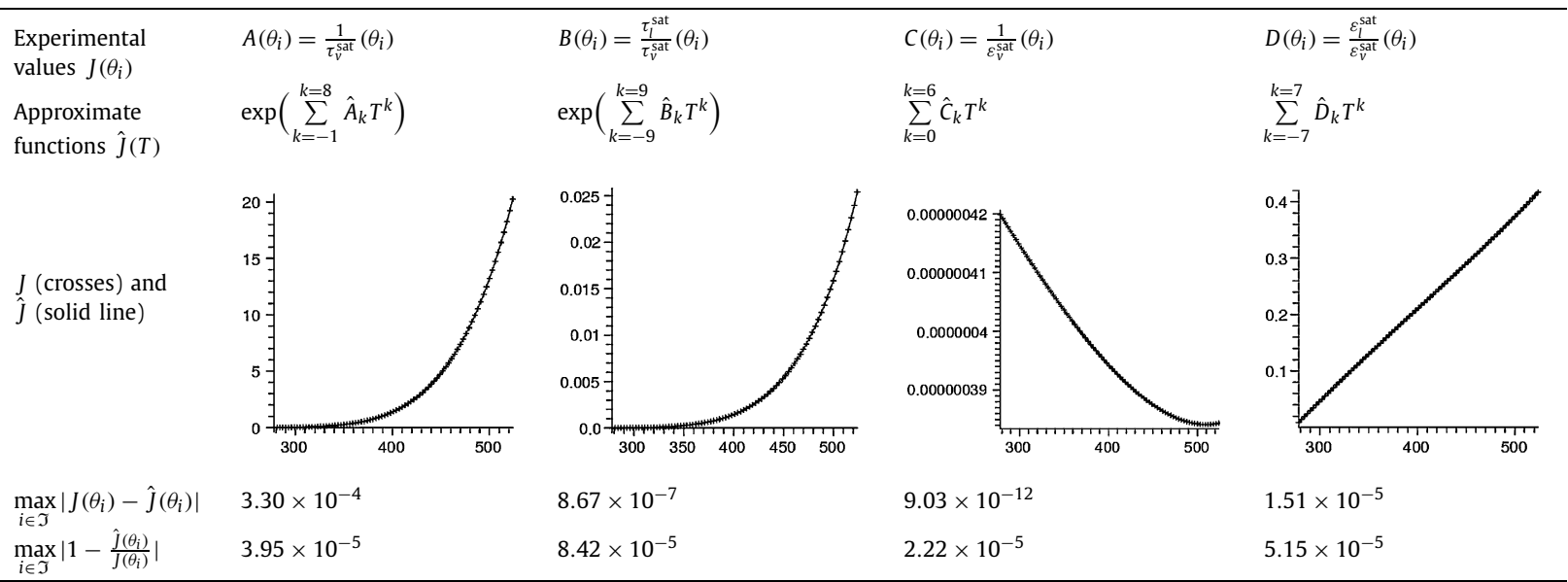

Fig. A.1. Graphs and errors of the functions $\hat{A}, \hat{B}, \hat{C}$ and $\hat{D}$ with respect to the experimental data $(A(\theta))_{\theta \in \mathfrak{T}},(B(\theta))_{\theta \in \mathfrak{T}},(C(\theta))_{\theta \in \mathfrak{T}},(D(\theta))_{\theta \in \mathfrak{T}}$.

\section{Appendix A. Example of approximate equilibrium for water liquid-vapor}

We use the same notation as in the previous section and all quantities are expressed in SI units. Let $\theta_{i}=278+3 i$, $i \in \mathfrak{I}=\{1, \ldots, 83\}$ be a discretization of the temperature interval [281,254]. We suppose that $T \mapsto(A, B, C, D)$ is given through a set of tabulated data $\left((A, B, C, D)\left(\theta_{i}\right)\right)_{i \in \mathfrak{I}}$ as in [12]. In order to define the approximate functions $\hat{A}, \hat{B}, \hat{C}, \hat{D}$, we simply use least squares approximation techniques over the set of discrete values $\left((A, B, C, D)\left(\theta_{i}\right)\right)_{i \in \mathfrak{I}}$. A simple and convenient choice of $\hat{A}, \hat{B}, \hat{C}, \hat{D}$ is provided by

$$
\hat{A}(T)=\exp \left(\sum_{k=-1}^{k=8} \hat{A}_{k} T^{k}\right), \quad \hat{B}(T)=\exp \left(\sum_{k=-9}^{k=9} \hat{B}_{k} T^{k}\right), \quad \hat{C}(T)=\sum_{k=0}^{k=6} \hat{C}_{k} T^{k}, \quad \hat{D}(T)=\sum_{k=-7}^{k=7} \hat{D}_{k} T^{k},
$$

where the coefficients $\hat{A}_{k}, \hat{B}_{k}, \hat{C}_{k}, \hat{D}_{k}$ are detailed in Table A.1. Errors and graphs of the functions $\hat{A}, \hat{B}, \hat{C}, \hat{D}$ are displayed in Fig. A.1. Let us measure the loss of accuracy caused by solving Eq. (7) instead of (6). We consider the discretization of the interval $[0,1]$ given by $y_{r}=(r-1) / 14, r \in \mathfrak{R}=\{1, \ldots, 15\}$. We define a discrete set of saturated two-phase states $(\tau, \varepsilon)_{(i, r)}$, $i \in \mathfrak{I}, r \in \mathfrak{R}$, by setting

$$
\tau_{(i, r)}=y_{r} \tau_{l}^{\text {sat }}\left(\theta_{i}\right)+\left(1-y_{r}\right) \tau_{\mathrm{v}}^{\text {sat }}\left(\theta_{i}\right), \quad \varepsilon_{(i, r)}=y_{r} \varepsilon_{l}^{\text {sat }}\left(\theta_{i}\right)+\left(1-y_{r}\right) \varepsilon_{\mathrm{v}}^{\text {sat }}\left(\theta_{i}\right), \quad \text { for } i \in \mathfrak{I}, r \in \Re .
$$

It is obvious that for each $(i, r) \in \mathfrak{I} \times \mathfrak{R}$, the exact solution of (6) with $\tau=\tau_{(i, r)}$ and $\varepsilon=\varepsilon_{(i, r)}$ is $T=\theta_{i}$. If we note $(\hat{y}, \hat{\theta})_{(i, r)}$ the solution obtained by approaching numerically with a $10^{-40}$ accuracy the solution of $(7)$ for $\tau=\tau_{(i, r)}$ and $\varepsilon=\varepsilon_{(i, r)}$, we observe that 


$$
\max _{\substack{i \in \mathfrak{I} \\ r \in \mathfrak{R}}}\left|\theta_{i}-\hat{T}_{(i, r)}\right|<6.72 \times 10^{-3} \mathrm{~K}, \quad \max _{\substack{i \in \mathfrak{I} \\ r \in \mathfrak{R}}}\left|1-\hat{T}_{(i, r)} / \theta_{i}\right|<1.29 \times 10^{-5}, \quad \max _{\substack{i \in \mathfrak{I} \\ r \in \mathfrak{R}}}\left|y_{r}-\hat{y}_{(i, r)}\right|<2.79 \times 10^{-5} .
$$

Consequently, for water liquid-vapor within the temperature range $T \in[281,524] \mathrm{K}$, solving the approximate equation (7) allows one to recover the value of the thermodynamical parameter at saturation with a good accuracy.

\section{References}

[1] G. Allaire, S. Clerc, S. Kokh, A five-equation model for the simulation of interfaces between compressible fluids, J. Comput. Phys. 181 (2) (2002) 577-616.

[2] G. Allaire, G. Faccanoni, S. Kokh, A strictly hyperbolic equilibrium phase transition model, C. R. Acad. Sci. Paris Ser. I 344 (2007) $135-140$.

[3] T. Barberon, Modélisation mathématique et numérique de la cavitation dans des écoulements multiphasiques compressibles, Ph.D. thesis, Université de Toulon et du Var, France, 2002.

[4] T. Barberon, Ph. Helluy, Finite volume simulations of cavitating flows, Comput. Fluids 34 (7) (2005) $832-858$.

[5] H.B. Callen, Thermodynamics and an Introduction to Thermostatistics, second edition, John Wiley \& Sons, 1985.

[6] G. Faccanoni, Étude d'un modèle fin de changement de phase liquide vapeur. Contribution à l'étude de la crise d'ébulition, Ph.D. thesis, École Polytechnique, France, 2008, http://pastel.paristech.org/4785/.

[7] G. Faccanoni, S. Kokh, G. Allaire, Numerical simulation with finite volume of dynamic liquid-vapor phase transition, in: Finite Volumes for Complex Applications, vol. V, ISTE and Wiley, 2008, pp. 391-398.

[8] Ph. Helluy, Quelques exemples de méthodes numériques récentes pour le calcul des écoulements multiphasiques, Mémoire d'habilitation à diriger des recherches, 2005.

[9] Ph. Helluy, N. Seguin, Relaxation models of phase transition flows, M2AN Math. Model. Numer. Anal. 40 (2) (2006) $331-352$.

[10] S. Jaouen, Étude mathématique et numérique de stabilité pour des modèles hydrodynamiques avec transition de phase, Ph.D. thesis, Université Paris 6 , France, 2001.

[11] O. Le Métayer, J. Massoni, R. Saurel, Élaboration des lois d'état d'un liquide et de sa vapeur pour les modèles d'écoulements diphasiques, Int. J. Thermal Sci. 43 (2003) 265-276.

[12] E. Lemmon, M. McLinden, D. Friend, Thermophysical Properties of Fluid Systems, National Institute of Standards and Technology, Gaithersburg, MD 20899, 2005, http://webbook.nist.gov.

[13] R. Menikoff, B. Plohr, The Riemann problem for fluid flow of real materials, Rev. Mod. Phys. 61 (1) (1989) $75-130$.

[14] A. Voß, Exact Riemann solution for the Euler equations with nonconvex and nonsmooth equation of state, Ph.D. thesis, Rheinisch-Westfälischen, 2005. 